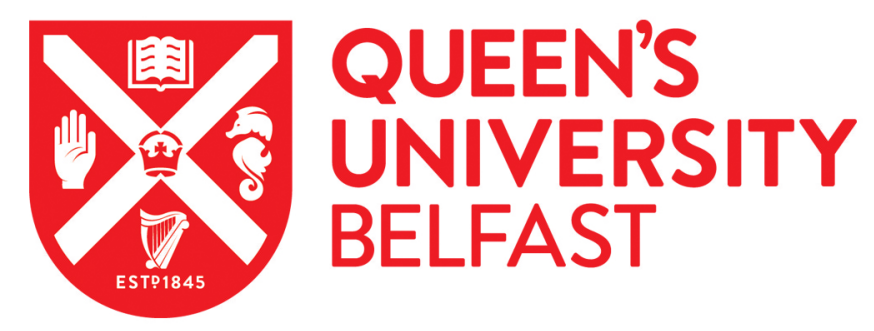

\title{
Transport and localization in single-walled carbon nanotubes
}

Fuhrer, M. S., Varadarajan, U., Holmes, W., Richards, P. L., Delaney, P., Louie, S. G., \& Zettl, A. (1998).

Transport and localization in single-walled carbon nanotubes. 69-73. Paper presented at Electronic Properties of Novel Materials: XII International Winter School, Kirchberg, Tyrol, Austria. https://doi.org/10.1063/1.56501

Queen's University Belfast - Research Portal:

Link to publication record in Queen's University Belfast Research Portal

\section{General rights}

Copyright for the publications made accessible via the Queen's University Belfast Research Portal is retained by the author(s) and / or other copyright owners and it is a condition of accessing these publications that users recognise and abide by the legal requirements associated with these rights.

Take down policy

The Research Portal is Queen's institutional repository that provides access to Queen's research output. Every effort has been made to ensure that content in the Research Portal does not infringe any person's rights, or applicable UK laws. If you discover content in the Research Portal that you believe breaches copyright or violates any law, please contact openaccess@qub.ac.uk. 


\title{
Transport and Localization in Single-Walled Carbon Nanotubes
}

\author{
M. S. Fuhrer, U. Varadarajan, W. Holmes, P. L. Richards, \\ P. Delaney, S. G. Louie, A. Zettl \\ Deparment of Physics, Universing of California. Berkeley, and Materials Sriences Division, Lawrence \\ Berkeley National Laboratorn, Berketey, CA 94720 USA
}

\begin{abstract}
We have measured the electrical transport properties of mats of single-walled carbon nanolubes (SWNT) as a function of applied electric and magnetic fjelds. We find that at low lemperatures the resistance as a function of temperature R(T) lollows the Motl variable range hopping (VRH) formula for hopping in three dimensions. Measurcment of the electric field dependence of the resistance $R(E)$ aliows for the determination of the Bohr radius of a localized slate a $\approx 650 \mathrm{~nm}$. The magnctoresistance (MR) of SWNT mal samples is Jarge and negative at all temperatures and fields studied, and can be qualitalively described by theories of MR for VRH systems. The Hall cocficient $R_{H}$ is positive and nearly temperalure-independent. The sign of $R_{H}$ agrees with the sign of the thermopower. The small magnilude of $\mathrm{R}_{\mathrm{H}}$ suggests a large carrier density, but may be the result of cancellation of electron and hole terms.
\end{abstract}

\section{Introduction}

The electrical transport properlies of single-walled carbon nanotubes are currently the subject of much debate. Individual achiral (armchair) SWNT are predicted to be melaltic $^{1-3}$. Resistance measurements on bulk samples (mats) of such tubes indeed show metallic behavior (positive $\mathrm{dR} / \mathrm{d} \mathrm{T}$ ) at high temperature, but negative $\mathrm{dR} / \mathrm{dT}$ at low temperature ${ }^{4}$. Transport measurements ${ }^{5,6}$ on individual tubes or bundles of tubes show some samples to be conducting to low temperatures, but the quantized electron energy levets which lead to such spectacular phenomena as Coulomb blockade cloud interpretation of the temperature dependence of the resistivity. It is then important to delemine whether the properties of the mat samples are intrinsic to the nanotubes or are a product of impurities or poor inter-lube connections.

The samples used in this sudy were produced by catalyst-assisted arcvalporization ${ }^{7}$ or laser-vaporization ${ }^{8}$ of a graphite source, yielding similar results. The resulting materiat was purified in $\mathrm{HNO}_{3}$ to remove catalyst impurities and amorphous carbon, and then washed and filtered. The samples are observed by transmission electron microscopy to consist of bundles of tens or hundreds of tubes arranged in a triangular lattice. The mats consist of a random network of these bundles, connected on a length sciale of approximately $100 \mathrm{~nm}$. Electrical contact to the bundles was made using silver paint in a standard four probe or Hall bar configuration. Sample resistances at room temperature ranged from 100 to $30 \% 0$ Ohms. 


\section{Nonlinear Resistance}

The $R(E)$ measurements used a standard pulsed current technique: $10 \mu$ sec current pulses were applied to the sample. The current and voltage were monitored simultaneously as a function of time using an oscilloscope, allowing the intrinsic resistance to be separated from (time-dependent) self-heating effects.

Figure 1 shows the R(T) behavior of a SWNT mat at various applied electric fields. The resistance of SWNT mats shows a striking dependence on E: a modest electric

field can completely suppress the resistance upturn and

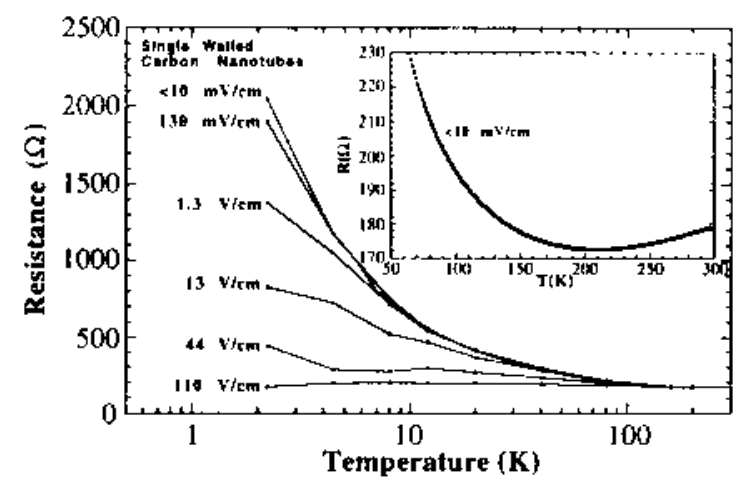

Figure 1. Resistance versus Temperature of SWNT mal at several applied electric fields. Inset shows high temperature $R(T)$ at low electric field.

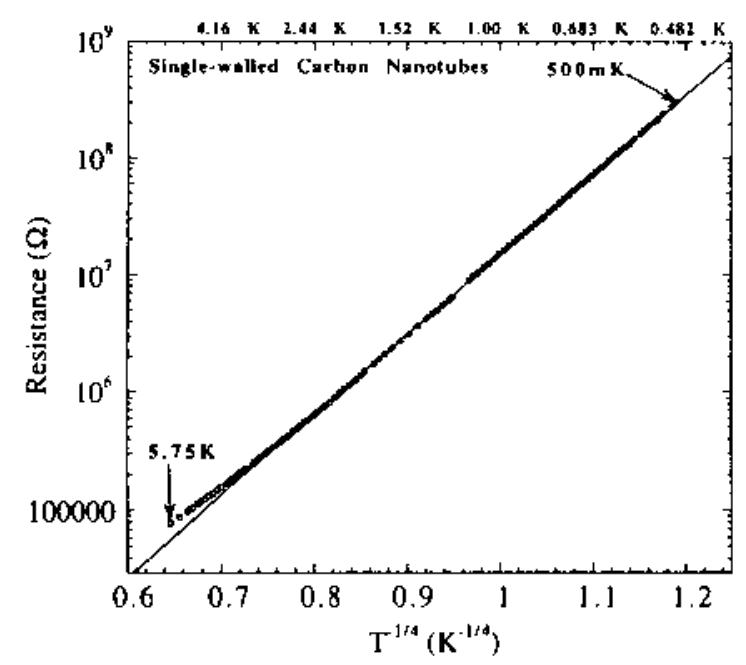

Figure 2. Resistance versus $\mathrm{T}^{-1 / 4}$ of SWNT mat showing three-dimensional variable range hopping. recover metallic behavior from $2.2 \mathrm{~K}$ to $300 \mathrm{~K}$. The inset of figure 1 shows the metallic high temperature resistance.

In order to clatify the nature of the non-linear resistance, measurements of $R(T)$ and $R(E)$ were repeated on the cold stage of an adjabatic demagnetization refrigerator, to temperatures as low as $227 \mathrm{mK}$. Figure 2 shows the low. temperature $R$ ( $T$ ) data plotted semi-logarithmically as a function of $\mathrm{T}^{-1 / 4}$; the data indicate $\mathrm{R} \alpha \exp \left(-\mathrm{T}^{1 / 4}\right)$, the Mott variable range hopping formula for three dimensions.

Temperature and electric ficid both have a similar delocalizing effect on the carriers. The two energy scales for temperature and electric field are $\mathrm{kT}$ and eEa, respectively, where $k$ is Boltzmann's constant, e the electronic charge, and a the Bohr radius of an electronic state. It is found that ${ }^{9}$.

$$
R(k T, 0)=\frac{R}{2}\left(0, \frac{3}{8} e E a\right),
$$

valid for finite values of $R$. Our $R$ (T) data are measured in the Ohmic regime, and thus give $\mathrm{R}(\mathrm{kT}, 0)$. We measured $\mathrm{R}(\mathrm{O}, \mathrm{E})$ at $227 \mathrm{mK}$. The data are plotted 
together in Figure 3. The temperature in Kelvin is scaled by the factor 3.5 in order to match the electric field in $\mathrm{V} / \mathrm{cm}$. The scaling of the two curves indicates $\mathrm{a} \approx 650 \mathrm{~nm}$.

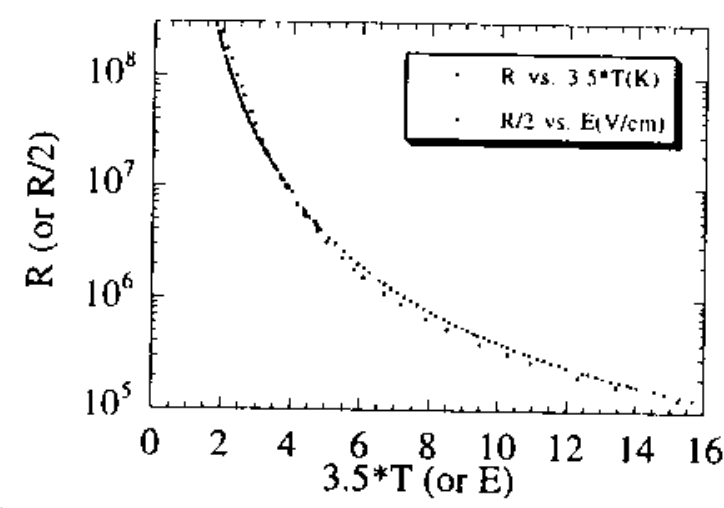

Figure 3. $R(E)$ at $227 \mathrm{mK}$ and $R(T)$ in the Ohmic regime for a SWNT mat. The scaling determines the radius of the localized states, $650 \mathrm{~nm}$.

SWNT mats are localized electronic systems where the electronic states are three-dimensional, with a radius of approximately $650 \mathrm{~nm}$, much greater than the average distance between bundle contacts observed by TEM. This result is inconsistent with any model of localization in which the electrons are confined to an individual tube or bundle. The dimensionality in Mott's VRH formula indicates that the number of available states for hopping is proportional to the distance $\mathrm{r}$ from the localized state raised to the d power. Hopping confined to a single tube or to nearest-neighbor bundles is one-dimensional, since the number of available states scales linearly with $r$ along the tube. Only if the localized state extended across many tube crossings would the number of available states be proportional to $\mathrm{r}^{3}$. We conclude that the localization is not due to poor junctions between tubes or bundles, but rather due to some intrinsic disorder, either in the individual tubes or bundles, or in the morphology of the network, which is inherently random.

\section{Magnetoresistance}

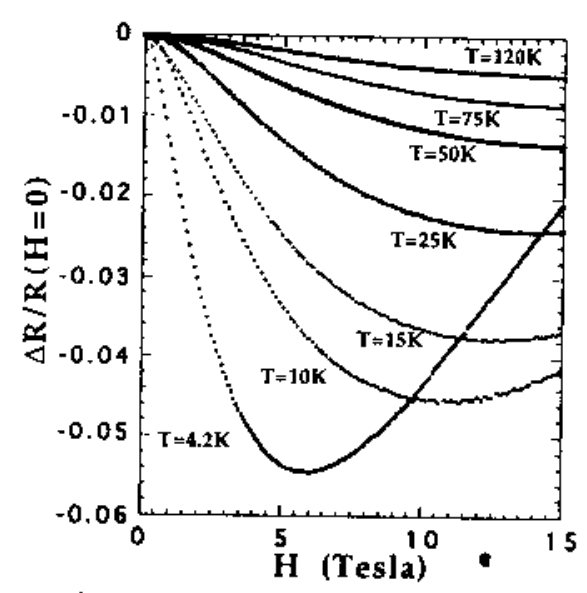

Figure 4. Magnetoresistance of a SWNT mat as a function of magnetic field at various temperatures.

different hopping paths between two states 10
Figure 4 shows the magnetoresistance (MR), defined as the change in resistance divided by the resistance at zero field, of a SWNT mat sample at various temperatures. The MR is negative at all fields and temperatures measured, but shows a minimum, increasing at higher fields.

Figure 5 shows the MR data replotted on a double logarithmic scale. The lines on the plot indicate $\mathrm{H}^{2}$ dependence of the MR, in agreement with the variable range hopping model. A tendency to saturation is also observed at high field, but the positive high field slope is unexplained.

In hopping systems, the effect of magnetic field can be modeled by considering the interference between all Magnetic fietd induces a phase difference 
in the different paths, which has a net delocalizing effect. In this model MR in variable range hopping systems is predicted to be negalive, with a quadratic dependence at low magnetic ficid, saturating to a constant value at high field.

\section{Hall Effect}

SWNT mat samples were prepared with a standard Hall bar geometry in order to measure the Hall effect. In order to circumvent the uncertainty in thickness of the SWNT films caused by their unusual morphology, the mass and area of each SWNT mat were measured. The experimentally accessible quantity is $\Delta \mathrm{R}_{x y} / \Delta \mathrm{H}$, the change in Hail resistance $\left(R_{x y}\right)$ with magnetic field. In a single band model,

$\frac{\Delta R_{x y}}{\Delta H}=\frac{R_{H}}{t}=\frac{1}{n e c}$

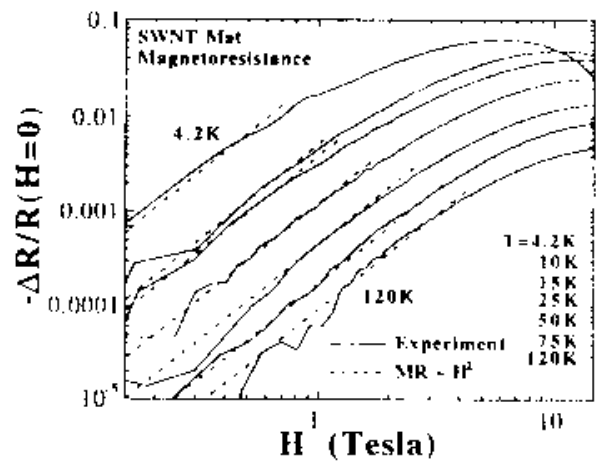

Figure 5. Negative magnetoresistance of a S $\mathrm{W} N \mathrm{NT}$ mat is plotted on a double-logarithmic scate. Sildid lines represent the experimental dala: straghlit delled lines indicate $\mathrm{H}^{2}$ behavior.

where $\mathrm{R}_{\mathrm{H}}$ is the Hall coefficient, $\mathrm{t}$ is the thickness of the sample, and $\mathrm{n}$ is the density of carriers per area. Knowing the mass per area of the film allows for a calculation of the number of carriers per carbon atom. Figure 6 shows $\mathrm{R}_{\mathrm{H}} / \mathrm{t}$ and $\mathrm{n}$ for a SWNT mal sample. The Hall coefficient is positive and nearly temperature independent. The positive sign of $\mathrm{R}_{\mathrm{H}} / \mathrm{t}$ agrees with the sign of the thermopower11. The magnitude of $\mathrm{R}_{\mathrm{H}} / \mathrm{t}$ indicates a carrier density of approximately 0.1 holes/atom in a single band picture. For a metallic $(10,10)$ tube this corresponds to about 4 holes per 40 atom unit cell, assuming all tubes in the sample are metallic, and contributing to the Hall effect. The presence of some percentage of semiconducting tubes would increase this number.

$$
\text { Band structure }
$$
calculations for the arnchair nanotubes show 2 states at the Fermi level, with an electronhole symmetry. The electron-

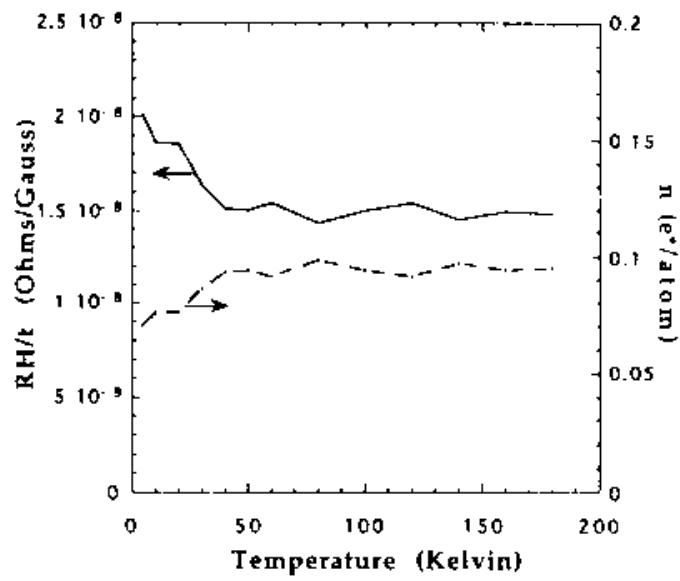

Figure 6. Hall coefficient divided by sample thickness and carrier density of SWNT mat plolled as a function of temperature. The solid line (Hall coefficient) corresponds to the left scale, the dotted line (carrier densily) corresponds to the right. 
hole symmetry should lead to zero Hall effect and thermopower, but significant positive Hall effect and thermopower are observed. Furthermore, the apparent $\mathbf{n}$ from the single hand picture is much too large. It has been predicted 12 that interactions between tubes in a bundle will significantly perturb the states near the Fermi level leading to the formation of electron-like and hole-like pockets of the Fermi surface, much as are found in graphite. We suggest that these electron and hole terms incompletely cancel, leading to a small $\mathrm{R}_{\mathrm{H}} /$, and apparent large $\mathrm{n}$.

We note that although a small downturn of the carrier density is observed at low temperatures, this cannot account for the large upturn in resistance. This is further evidence that the resistance upturn is a reduction of the mobility of the carriers, as would be expected in the case of localization, not in their number, as would be the case if a gap in the Fermi surface were causing the resistance upturn.

\section{Conclusions}

We have measured the electrical transport properties of SWNT mats in electric and magnetic fields. Although composed of one-dimensional wires, mats of single-walled catrbon nanotubes exhibit three-dimensional metallic behavior, as evidenced by their metallic resistivity and the Hall effect. At low temperatures, the carriers are localized, leading to a 3D variable range hopping form of the resistance. Comparison of the temperature and electric field dependencies of the resistance allows for the extraction of the Bohr radius of localized states $a=650 \mathrm{~nm}$, consistent with three-dimensional states. The magnetoresistance of SWNT mats is consistent with theories of MR for variable range hopping, although the high-field positive component of the MR remains unexplained. The Hall effect is positive in agreement with TEP results and small in magnitude, which would indicate an unusually large hole concentration in a single band model. The small Hall coefficient likely results from the cancellation of electron and hole terms, supporting the idea that tube-tube interactions in a bundle perturb the Fermi surface significantly.

We thank Prof. R. E. Smalley for providing some of the samples used in this study. This work was supported in part by the Director, Office of Energy Research, Office of Basic Energy Sciences, Materials Sciences Division of the U. S. Department of Energy under Contract No. DE-AC03-76SF00098, and by the National Science Foundation, Grant No. DMR95-20554.

\section{References}

1. J. W. Mintmire, B. I. Dunlap and C. T. White, Phys. Rev. Lett. 68, 631 (1992).

2. N. Hamada, S. Sawada and A. Oshiyama, Phys. Rev. Lett. 68, 1579 (1992).

3. R. Sailo, M. Fujita, G. Dresselhaus and M. S. Dresselhaus, Appl. Phys. Lett. 60, 2204 (1992).

4. J. E. Fischer, et al., Phys. Rev. B 55, R492l (1997).

5. S. J. Tans, et al., Nature 386, 474 (1997).

6. M. Bockrath, et al., Science 275, 1922 (1997).

7. C. Jөimet, et al., Nature 388, 756 (1997)

8. A. Thess, et al., Science 273, 483 (1996).

9. N. Apsiey and H. P. Hughes, Philosophical Magazine 31, 1327 (1975).

10. U. Sivan, O. Entin-Wohlman and Y. Jmry, Phys. Rev. Lett. 60, 1566 (1988).

II. J. Hone, et al., Physical Review Letters 80, 1042 (1997).

12. P. Delaney, H. J. Choi, J. Ihm, S. G. Louie and M. L. Cohen, Nature 391, 466 (1998). 